\title{
Do Personality Traits Modulate the Effect of Emotional Visual Stimuli on Auditory Information Processing?
}

\author{
Solange Mardaga and Michel Hansenne
}

Department of Cognitive Sciences, University of Liège, Belgium

\begin{abstract}
Several lines of evidence attest robust relationships between personality dimensions and emotions, including cognitive aspect of emotion. More particularly, many studies reported strong relationships between extraversion, the behavioral activation system (BAS), and the cognitive processing of positive information, on the one hand, and between neuroticism, the behavioral inhibition system (BIS), and the processing of negative information, on the other hand. Recently, DePascalis, Awari, Matteucci, and Mazzocco (2005) reported that personality traits modulated the effect of the emotional visual stimuli on the mismatch negativity (MMN). The aim of the present study was to replicate these data and extend them to other personality dimensions. Auditory MMN was recorded in normal subjects simultaneously to the presentation of emotional pictures selected as neutral, positive, or negative from the International Affective Picture System, and presented in randomized order. The results support the recent finding that personality (namely, BIS and harm avoidance) modulates the influence of emotional (negative) context on auditory information processing. The present findings suggest that the modulation by personality of change detection in the unattended environment as a function of context valence is limited to unpleasant context.
\end{abstract}

Keywords: personality, emotions, EPRs, mismatch negativity

\section{Introduction}

The existence of relations between personality and emotions is well documented, especially between extraversion, the Behavioral Activation System (BAS) and positive affects, as well as between neuroticism, the Behavioral Inhibition System (BIS), and negative affects (e.g., Canli, Sivers, Withfield, Gotlib, \& Gabrieli, 2002; Corr, 2002; Gable, Reis, \& Elliot, 2000; Gross, Sutton, \& Ketelaar, 1998; Heponiemi, Keltikangas-Järvinen, Puttonen, \& Ravaja, 2003). Many studies have reported that personality consistently modulates the interactions between emotion and cognition (Bradley \& Mogg, 1994; Gomez \& Gomez, 2002; Rusting, 1999). For instance, Gomez and Gomez (2002) found that high-BIS subjects generated more negative words in a word-fragmentation task, recognized more negative words in a word-recognition task, and recalled more negative words in a free word-recall task than low-BIS subjects, whereas high-BAS subjects generated, recognized, and recalled more positive words in the three tasks than low-BAS subjects. These results indicate that BIS is mainly associated with the processing of unpleasant information, while BAS is mainly associated with the processing of pleasant information, and more generally, that cognitive processing of emotional information is modulated by personality.

Event-related potentials (ERPs) are modifications of the cerebral electric activity that reflect diverse aspects of cog- nitive processes; they constitute a useful tool to investigate cognition (e.g., attentional processes, memory, language) in normal as well as in psychopathological subjects (Fabiani, Gratton, \& Coles, 2000). One of the most extensively studied ERPs is the mismatch negativity (MMN; Näätänen, Gaillard, \& Mantysalo, 1978), a negative fronto-central component of the auditory ERP with late latency (usually peaking around $200 \mathrm{~ms}$ ) that is linked to a switch reflecting attention to physical environment change (Näätänen, 2001; Picton, Alain, Otten, Ritter, \& Achim, 2000). It is generally elicited by a deviant, low-probability stimulus (e.g., a change in the frequency, intensity, or localization of the tone) in a sequence of standard auditory stimuli. The MMN is presumably generated by a mismatch process between the sensory input from a deviant stimulus and a short-term memory (sensory-memory) trace representing the physical features of the standard stimuli; this process appears to be automatic since the MMN is elicited even by changes in unattended auditory stimuli (Näätänen, 2000, 2001). Recent findings have also suggested that the transient auditory sensory-memory representation underlying the $\mathrm{MMN}$ is facilitated by a long-term memory representation of the corresponding stimulus (Winkler, Cowan, Csépé, Czigler, \& Näätänen, 1996). This means that some characteristics of the sensory-memory traces involved in the elicitation of this component are stored in a more durable representation and that these memory traces can be reactivated (Winkler \& Cowan, 2005). 
ERPs can be viewed as an interesting approach to explore the influence of emotions on cognition. Several studies reported that P300 amplitude is modulated by the emotional character of information (Amrhein, Mühlberger, Pauli, \& Wiedemann, 2004; Delplanque, Silvert, Hot, \& Sequeira, 2006; Palomba, Angrilli, \& Mini, 1997). For instance, Delplanque et al. (2006), within a three-stimulus, visual, oddball protocol with emotional pictures as the novel stimuli, reported an increase of P300 amplitude for pleasant and unpleasant stimuli relative to neutral novel ones. This indicates that the memory updating process indexed by P300 is influenced by the emotional valence and arousal characteristics of information. Some studies also suggested that personality traits could modulate the emotional effects on ERPs (DePascalis \& Speranza, 2000; DePascalis, Strippoli, Riccardi, \& Vergari, 2004). DePascalis et al. (2004) reported that anxious (high-BIS) subjects showed larger P300 amplitudes to unpleasant targets than nonanxious subjects, which is consistent with the above-reported relation between BIS and processing of negative information.

Few studies have investigated the effect of emotional visual stimuli on the MMN. Surakka, Tenhunen-Eskelinen, Hietanen, and Sams (1998) recorded auditory MMN simultaneously to the presentation of emotional pictures (neutral, positive, and negative). They reported smaller MMN amplitudes recorded during the presentation of the positive stimuli as compared to MMNs recorded during the presentation of neutral and negative stimuli; indicating that a positive, low-arousing context reduces vigilance toward the environment. Recently, DePascalis, Awari, Matteucci, and Mazzocco (2005), with the same protocol as Surakka et al. (1998), found that MMN amplitudes were higher during the presentation of negative stimuli as compared to neutral and positive ones, reflecting increased vigilance within a negative emotional context. Taken together, the results of these two studies suggest that specific valence and arousal characteristics of a visual environment could act as biological signals of either high or low danger, and consequently increased or reduced need for vigilance to physical environment change, reflected here by the MMN. Moreover, DePascalis et al. (2005) reported that personality traits influenced the effect of the emotional visual stimuli on the MMN. They found that high-BIS/low-BAS subjects were the most sensitive, and low-BIS/high-BAS subjects the least sensitive to the negative stimulations, indicating that both BIS and BAS modulated sensitivity to unpleasant pictures. Interestingly, these results indicate that personality differences in emotional sensitivity modify the earlier stages of information processing.

The aim of the present study was to replicate and extend the above findings showing that personality traits influence the effect of emotional visual stimuli on automatic information processing with the Cloninger's biosocial model. This model posits four innate temperaments: novelty seeking (NS), harm avoidance (HA), reward dependence, and persistence; and three acquired characters: self-directedness, cooperativeness, and self-transcendence (Cloninger, 1987; Cloninger, Svrakic, \& Przybeck, 1993). Particularly interesting in the present context, NS is defined as the tendency to respond actively to novel stimuli leading to pursuit of rewards and escape from punishment, and high-NS subjects would respond intensely to cues for potential rewards; HA is defined as the tendency to inhibit responses to signals of aversive stimuli that lead to avoidance of punishment and nonreward, and high-HA subjects would respond intensely to signals of aversive stimuli (Cloninger, 1987). We, thus, expected that high-NS subjects would be more sensitive to the positive context influence, while high-HA subjects would be more sensitive to the negative context influence.

\section{Methods}

\section{Subjects}

The study was conducted on a sample of 60 participants (28 males) aged between 18 and 35 years (mean $=22.58$, $S D=3.64$ ). They were naïve to the aim of the experiment and to the pictures used in the protocol. All had normal or corrected to normal vision. The Ethical Committee of the University of Liège Psychology School approved the protocol and all subjects gave their informed consent to take part to the study.

The participants completed the French version of the revised form of the Temperament and Character Inventory (TCI-R; Cloninger, 1999), the Neuroticism Extraversion Openness Personality Inventory (NEO-PI-R; Costa \& McCrae, 1990), the BIS/BAS scales (Carver \& White, 1994), and the Sensitivity to Punishment and Sensitivity to Reward Questionnaire (SPSRQ; Torrubia, Avila, Moltó, \& Caseras, 2001).

\section{Procedure}

Auditory MMN was recorded simultaneously to the presentation of visual emotional stimuli. The emotional stimuli consisted of 90 colored pictures ${ }^{1}$ selected as neutral, pleasant, and unpleasant (30 in each category) from the IAPS (International Affective Picture System; Center for

\footnotetext{
1 Neutral pictures: 1121, 1302, 1321, 1390, 1560, 1603, 1811, 1942, 2000, 2160, 2220, 2480, 2500, 2580, 5470, 5629, 5920, 5940, 5950, 7620, 7640, 8030, 8041, 8190, 8190, 8200, 8370, 9190, 9411, 9582; pleasant pictures: 1463, 1710, 1722, 1920, 2050, 2170, 2208, 2209, $2303,2345,2352,2550,2620,4607,4610,4611,4640,4651,4659,4660,4666,4670,4672,4680,7325,8470,8501$, for women: 4520, 4640, 4658, for men: 4180, 4220, 4290; unpleasant pictures: 1200, 2141, 3071, 3110, 3180, 3230, 3301,3530, 3550, 6020, 6230, 6550, 6570, 6831, 6940, 8230, 9090, 9102, 9120, 9230, 9402, 9420, 9430, 9433, 9560, 9561, 9570, 9630, 9810, 9910.
} 
Table 1. Mean, median, and SD for all personality dimensions assessed as well as Pearson's correlations between personality dimensions (correlations in bold are significant at $p<.05$ threshold)

\begin{tabular}{|c|c|c|c|c|c|c|c|c|c|c|c|c|c|c|c|c|}
\hline & NS & HA & $\mathrm{RD}$ & $\mathrm{P}$ & SD & Coop & ST & BIS & BAS & $\mathrm{N}$ & $\mathrm{E}$ & $\mathrm{O}$ & A & Con & SP & SR \\
\hline Mean & 34.02 & 93.53 & 114.14 & 121.49 & 120.05 & 108.29 & 10.33 & 9.65 & 34.02 & 93.53 & 114.14 & 121.49 & 120.05 & 108.29 & 10.33 & 9.65 \\
\hline Median & 103.50 & 92.00 & 105.00 & 111.50 & 139.00 & 136.00 & 72.00 & 21.00 & 39.00 & 91.00 & 114.00 & 124.00 & 117.00 & 109.00 & 10.00 & 9.50 \\
\hline SD & 12.27 & 19.08 & 17.56 & 15.94 & 18.03 & 17.45 & 4.94 & 3.82 & 12.27 & 19.08 & 17.56 & 15.94 & 18.03 & 17.45 & 4.94 & 3.82 \\
\hline NS & 1.00 & -0.16 & 0.19 & -0.26 & 0.02 & -0.06 & -0.04 & -0.15 & 0.22 & 0.04 & 0.36 & 0.18 & -0.26 & -0.35 & -0.18 & 0.06 \\
\hline HA & -0.16 & 1.00 & -0.10 & -0.16 & -0.39 & -0.07 & 0.02 & 0.68 & -0.15 & 0.69 & -0.38 & -0.10 & 0.11 & -0.16 & 0.77 & 0.15 \\
\hline $\mathrm{RD}$ & 0.19 & -0.10 & 1.00 & 0.06 & 0.36 & 0.51 & 0.14 & 0.22 & 0.04 & 0.09 & 0.49 & 0.09 & 0.42 & 0.05 & -0.19 & 0.04 \\
\hline $\mathrm{P}$ & -0.26 & -0.16 & 0.06 & 1.00 & 0.22 & -0.05 & 0.15 & 0.06 & 0.22 & 0.07 & 0.34 & 0.04 & -0.13 & 0.80 & -0.18 & 0.16 \\
\hline SD & 0.02 & -0.39 & 0.36 & 0.22 & 1.00 & 0.38 & -0.12 & -0.15 & 0.00 & -0.45 & 0.38 & -0.06 & 0.28 & 0.47 & -0.44 & -0.34 \\
\hline $\mathrm{C}$ & -0.06 & -0.07 & 0.51 & -0.05 & 0.38 & 1.00 & 0.29 & 0.14 & -0.23 & -0.06 & 0.10 & 0.27 & 0.75 & -0.06 & -0.05 & -0.29 \\
\hline ST & -0.04 & 0.02 & 0.14 & 0.15 & -0.12 & 0.29 & 1.00 & -0.08 & -0.20 & 0.05 & -0.00 & 0.43 & 0.17 & 0.02 & -0.09 & 0.03 \\
\hline BIS & -0.15 & 0.68 & 0.22 & 0.06 & -0.15 & 0.14 & -0.08 & 1.00 & -0.16 & 0.65 & -0.11 & -0.10 & 0.21 & -0.00 & 0.56 & 0.13 \\
\hline BAS & 0.22 & -0.15 & 0.04 & 0.22 & 0.00 & -0.23 & -0.20 & -0.16 & 1.00 & 0.00 & 0.21 & -0.09 & -0.18 & 0.13 & -0.19 & 0.10 \\
\hline $\mathrm{N}$ & 0.04 & 0.69 & 0.09 & 0.07 & -0.45 & -0.06 & 0.05 & 0.65 & 0.00 & 1.00 & -0.10 & 0.07 & -0.14 & -0.15 & 0.63 & 0.34 \\
\hline $\mathrm{E}$ & 0.36 & -0.38 & 0.49 & 0.34 & 0.38 & 0.10 & -0.00 & -0.11 & 0.21 & -0.10 & 1.00 & 0.23 & -0.02 & 0.38 & -0.46 & 0.21 \\
\hline $\mathrm{O}$ & 0.18 & -0.10 & 0.09 & 0.04 & -0.06 & 0.27 & 0.43 & -0.10 & -0.09 & 0.07 & 0.23 & 1.00 & -0.08 & -0.11 & -0.13 & 0.28 \\
\hline A & -0.26 & 0.11 & 0.42 & -0.13 & 0.28 & 0.75 & 0.17 & 0.21 & -0.18 & -0.14 & -0.02 & -0.08 & 1.00 & -0.06 & 0.14 & -0.38 \\
\hline $\mathrm{C}$ & -0.35 & -0.16 & 0.05 & 0.80 & 0.47 & -0.06 & 0.02 & -0.00 & 0.13 & -0.15 & 0.38 & -0.11 & -0.06 & 1.00 & -0.21 & -0.03 \\
\hline SP & -0.18 & 0.77 & -0.19 & -0.18 & -0.44 & -0.05 & -0.09 & 0.56 & -0.19 & 0.63 & -0.46 & -0.13 & 0.14 & -0.21 & 1.00 & 0.15 \\
\hline SR & 0.06 & 0.15 & 0.04 & 0.16 & -0.34 & -0.29 & 0.03 & 0.13 & 0.10 & 0.34 & 0.21 & 0.28 & -0.38 & -0.03 & 0.15 & 1.00 \\
\hline
\end{tabular}

$\mathrm{NS}=$ novelty seeking, $\mathrm{HA}=$ harm avoidance, $\mathrm{RD}=$ reward dependence, $\mathrm{P}=$ persistence, $\mathrm{SD}=$ self-directedness, Coop = cooperation, $\mathrm{ST}=$ self-transcendence, BIS and BAS = Carver \& White scales for behavioral activation and inhibition system, $\mathrm{N}=$ neuroticism, $\mathrm{E}=$ extraversion, $\mathrm{O}=$ openness, $\mathrm{A}=$ agreeableness, $\mathrm{Con}=$ consciousness, $\mathrm{SP}=$ sensitivity to reward, $\mathrm{SR}=$ sensitivity to punishment.

the Study of Emotion and Attention, 1999). The slides were displayed in randomized order during $18 \mathrm{~s}$, and followed by a 5 -s black screen on a 16-inch computer screen (1400 $\times 1050$ pixels, refresh rate $60 \mathrm{~Hz}$ ) at a distance of approximately $1 \mathrm{~m}$ from the participants.

At their arrival, the participants completed the personality questionnaires, and the cap containing the electrodes was applied. The recording session consisted of three blocks of $11 \mathrm{~min}$. After each MMN recording session, the participants were presented once again with the same pictures and were asked to rate pleasure and emotional arousal for each picture (the rating task lasted about $10 \mathrm{~min}$ ). The rating scales for picture pleasure and arousal were 9-point (1-9) Likert scales, ranging from $1=$ unpleasant to $9=$ pleasant and from $1=$ calm to $9=$ arousing, respectively.

\section{ERP Recording and Data Analysis}

ERP recording was conducted while the participants were comfortably seated in a sound-attenuated room. The MMN was elicited by an auditory, passive, oddball protocol with $80 \%$ standard stimuli $(1000 \mathrm{~Hz}, 70 \mathrm{~dB}, 40 \mathrm{~ms}$ duration) and $20 \%$ deviant stimuli ( $1100 \mathrm{~Hz}, 70 \mathrm{~dB}, 40 \mathrm{~ms}$ duration), presented in a pseudo-randomized order, so that at least two frequent tones preceded a rare one. A total of 2070 acoustic stimulations was presented binaurally via headphones at the rate of one trial every second.
The EEG activity was recorded at three sites of the 10-20 system (Fz, Cz, Pz), using Ag/AgCl electrodes, mastoid for reference, and forehead for ground. All sites were cleaned with acetone and abraded before recording to maintain a resistance below $5 \mathrm{k} \Omega$. EOG was recorded from above and beside the left eye. Amplifier gains were set at 20 , with a band pass of $0.05-35 \mathrm{~Hz}$. The EEG was recorded during $900 \mathrm{~ms}$ with a $150 \mathrm{~ms}$ prestimulus baseline. Trials on which the EEG or EOG exceeded $30 S D$ of the voltage distribution were rejected automatically (Van de Velde, Ghosh, \& Cluitmans, 1999). There were no other rejection criterion, and individual curves were not systematically inspected visually for artifacts. MMN was defined as the highest negative peak between 100 and 200 ms after stimulus onset in the difference-wave obtained by subtracting frequent-tone from deviant-tone waves.

\section{Statistical Analysis}

All the statistical analyses were performed with Statistica (6.0) for Windows. The effect of picture categories (neutral, positive, and negative) on pleasure and arousal ratings were analyzed with Friedman ANOVAs, with Wilcoxon test used for paired comparisons. The influence of picture categories (neutral, positive, and negative) on MMN latencies and amplitudes were analyzed with two-way repeatedmeasure ANOVAs ( 3 picture categories $\times 3$ electrode loca- 

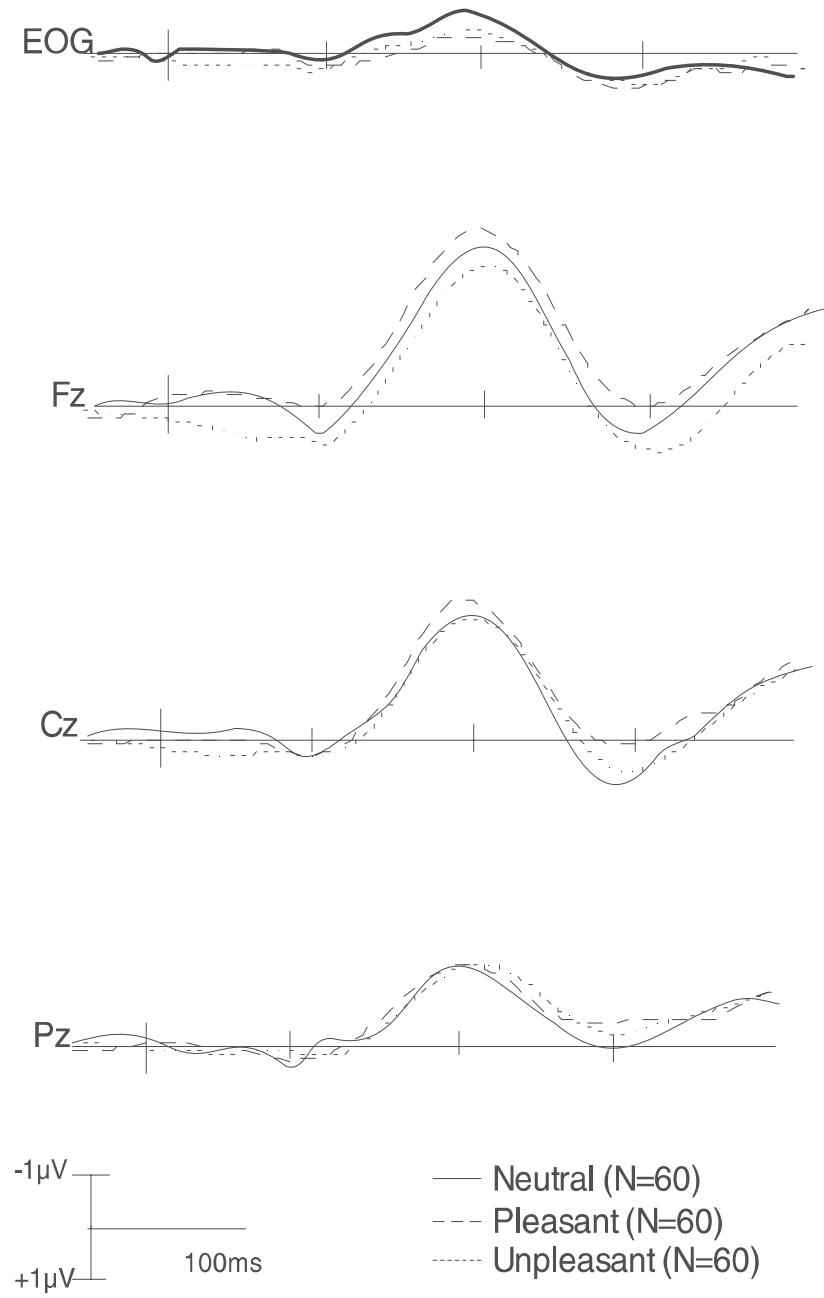

Figure 1. Grand average MMN difference waves recorded while subjects were presented neutral, positive, and negative pictures.

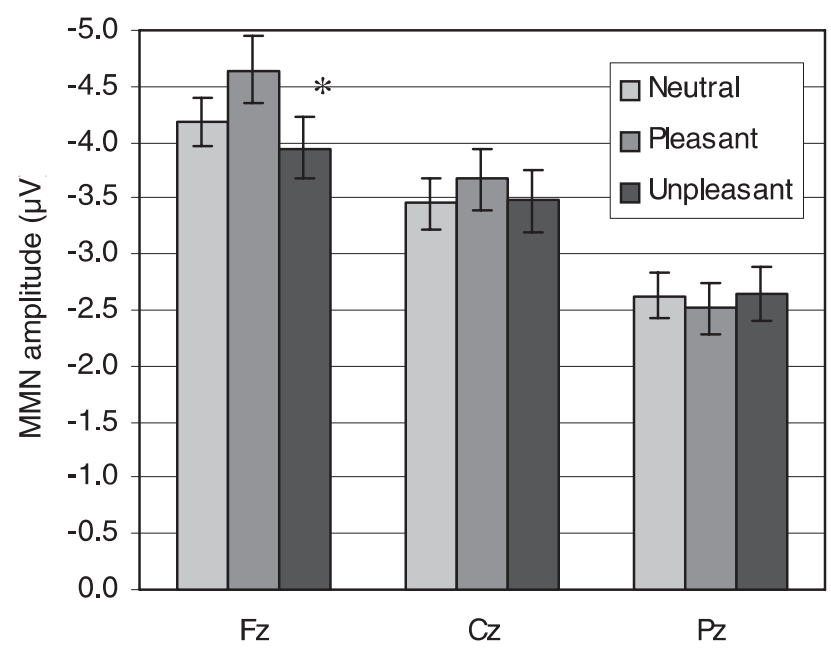

Figure 2. Mean MMN amplitude recorded within neutral, positive, and negative emotional contexts, at the three recording sites $(\mathrm{Fz}, \mathrm{Cz}$, and $\mathrm{Pz})$. * indicates a significant difference at $p<.05$ compared to pleasant pictures.

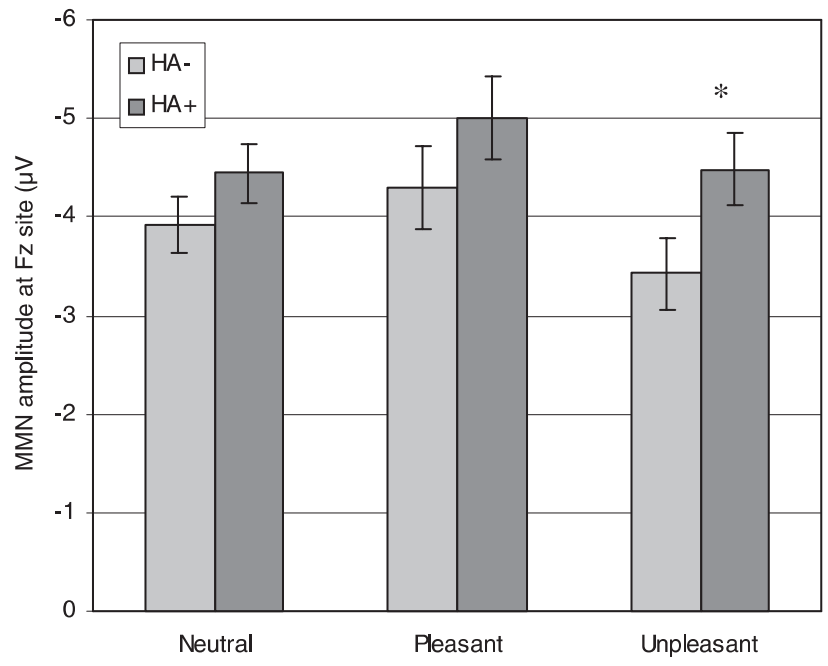

Figure 3. Mean MMN amplitude recorded within neutral, positive, and negative contexts in low- and high-HA groups at Fz location. $*$ indicates a significant difference at $p<.05$ compared to low-HA subjects.

tions). In order to investigate interactions between MMN and personality data, participants were assigned to groups on the basis of high and low scores for each personality dimension expected to modulate emotional responsivity (namely NS, HA, BIS, BAS, SP, SR, E, and N), with highand low-subjects selected, respectively, over and below the median of the score distribution. Three-way repeated-measure ANOVAs with 3 picture categories $\times 3$ electrode locations $\times 2$ personality groups were performed, with picture categories and electrode locations as within-subject factors. For all ANOVAs, the Greenhouse-Geisser correction was applied to significance levels for effects including a repeated-measure factor, and Student's $t$ tests were used as post hoc analysis.

\section{Results}

The mean, median, and $S D$ for all personality dimensions assessed, as well as Pearson's correlations between personality dimensions, are depicted in Table 1.

Mean pleasure values for neutral, positive, and negative pictures were $5.3,6.2$, and 2.7 , respectively, and mean arousal values were 4.7, 5.2, and 5.8. With pleasure rating as the dependent variable, a Friedman ANOVA showed a significant effect of picture category, $\chi^{2}{ }_{(2)}=107.32, p<$ .001. Paired comparisons showed significant differences between all picture categories (neutral-positive: $T=129.5$, neutral-negative: $T<.001$, positive-negative: $T<.001$; all $p$ values $<.001)$. With arousal rating as the dependent variable, a Friedman ANOVA showed a significant effect of picture category, $\chi^{2}{ }_{(2)}=42.44, p<.001$. Paired comparisons showed significant differences between all picture cat- 
egories (neutral-positive: $T=301.5$, neutral-negative: $T=$ 169.5, all $p$ values < .001; positive-negative: $T=548, p=$ $.007)$.

The great average curves recorded during the presentation of the three kinds of visual stimuli are depicted in Figure 1 . The two-way repeated-measure ANOVA (3 picture categories $\times 3$ electrode locations) conducted with MMN amplitude as the dependent variable revealed a main effect of electrode location, $F(2,118)=118.65, p<.001, \eta^{2}=$ 0.67 , and a significant Electrode $\times$ Picture Category interaction, $F(4,236)=3.66, p<.01, \eta^{2}=0.06$. Paired comparisons showed that MMN amplitudes recorded in a pleasant context were larger than in an unpleasant context, at frontal sites only, $t_{(59)}=2.08, p=.04$ (Figure 2).

The three-way ANOVAs revealed a main effect of NS, $F(1,58)=5.22, p=.03, \eta^{2}=0.08$, reflecting that high-NS subjects show larger MMN amplitudes than low-NS subjects, and a marginal effect of $\mathrm{HA}, F(1,58)=3.75, p=.06$, $\eta^{2}=0.06$, reflecting that high-HA subjects show larger MMN amplitudes than low-HA subjects. A marginal BIS $\times$ Picture Category $\times$ Electrode interaction appeared, $F(4,192)=2.61, p=.058, \eta^{2}=0.05$, reflecting that highBIS subjects showed larger MMN amplitude at frontal locations within unpleasant context than low-BIS subjects, $t_{(48)}=2.04, p=.047$, but not within neutral or pleasant context, $t_{(48)}=0.82, p=.42$ and $\mathrm{t}_{(48)}=0.78, p=.44$, respectively. After Greenhouse-Geisser correction, the HA $\times$ Picture Category $\times$ Electrode interaction did not reach significance, $F(4,232)=1.88, p=.14, \eta^{2}=0.03$, but because specific expectations had been formulated regarding this dimension, HA groups were compared regarding MMN amplitude observed in different emotional contexts, and high-HA subjects were found to show larger MMN amplitude at frontal locations within unpleasant context than low-HA subjects, $t_{(58)}=2.03, p=.047$, but not within neutral or pleasant context, $t_{(58)}=1.25, p=.22$ and $t_{(58)}=1.22$, $p=.23$, respectively (see Figure 3 ).

No effect reached statistical significance for either twoway or three-way ANOVAs performed with MMN latency as the dependent variable.

\section{Discussion}

The results of this study showed that pleasant contexts elicited larger MMN than unpleasant contexts, reflecting more automatic attentional allocation to the unattended environment when the visual context has a positive valence. This suggests that a pleasant context increased the perceived need for change detection, and, thus, vigilance in the subjects. This result diverges from previous results reported either by Surakka et al. (1998) (smaller MMN within pleasant context) or DePascalis et al. (2005) (larger MMN within unpleasant context). One point that can be advanced to explain this divergence is the difference between the emotional arousal of pictures presented in the present study and in the previous ones. Thus, the pleasant pictures presented here were rated as more arousing than those presented by Surakka et al. (1998) (i.e., 4), and the perception of a positive context as requiring less vigilance might be limited to low-arousing, but not high-arousing pleasant context; indeed, whereas positive, low-arousing contexts may be a sign of low danger, positive high-arousing contexts may evoke potential reward and, therefore, induce increased vigilance. As well, in the present study, neutral and negative picture ratings were quite close, while they were highly dissimilar in the DePascalis et al. study (2.9 and 6.4); so increased MMN amplitude might be observable only in negative, high-arousing contexts. In summary, it could be argued that the influence of the emotional context on MMN amplitude might be closely related to the emotional arousal resulting from the context, and not only to its valence. Indeed, arousal characteristics of the stimuli on emotional responses has previously been reported as highly relevant by studies using other psychophysiological measures of emotions such as skin-conductance response (Lang, Greenwald, Bradley, \& Hamm, 1993). On the other hand, it should be noted that Surakka et al. (1998) reported no difference between MMN amplitudes obtained during the presentation of neutral and negative stimuli, in spite of quite dissimilar arousal ratings (3.5 and 7.1, respectively). Thus, although methodological differences may be advanced to explain why the present study did not replicate previous findings, divergent results reported in previous studies suggest that the influence of emotional context on auditory information processing should be considered carefully before further replication.

The present results also revealed personality influences on auditory MMN amplitudes, regardless of emotional context. Namely, high-HA and high-NS subjects showed larger MMN than low-HA subjects and low-NS subjects, respectively. Several studies previously reported relationships between MMN amplitude and personality dimensions such as HA (Hansenne et al., 2003), neuroticism (Wang, Zhu, Pan, Hu, \& Wang, 2001), impulsivity (Franken, Nijs, \& VanStrier, 2005), or extraversion (Sasaki, Campbell, Gordon Bazana, \& Stelmack, 2000). The reason why both BIS- and BAS-related dimensions were occasionally reported to be related to MMN amplitude has still to be clarified, but these results suggest that, asides from emotional differences, personality may influence information cognitive processing, giving support to the notion of personality-related attentional styles.

Finally, personality modulation of the emotional context influence was reported here. Consistent with DePascalis et al. (2005) results, only the negative context influence was modulated by personality, namely HA and BIS scales in the present study and SP/SR scales in the DePascalis study. This result suggests that, although unpleasant context did not elicit increased vigilance per se, it allows the detection of different attentional attitudes across subjects: Anxious subjects (i.e., high-HA and high-BIS subjects) would keep a higher level of attention toward the unattended environ- 
ment, within unpleasant contexts, than low-anxious subjects, reflecting a consistent defensive, danger-expecting, attitude (Cloninger et al., 1993). This is also consistent with the previously reported higher sensitivity to unpleasant stimuli and a preferential processing of unpleasant material in high BIS-related subjects (e.g., Corr, 2002; Gable et al., 2000; Gomez \& Gomez, 2002; Rusting, 1999).

It should be noted that a second experiment conducted in the laboratory (unpublished data) aimed to replicate previous findings with emotional pictures presented, not in randomized order, but by blocks (i.e., pictures from the same category - neutral, pleasant, or unpleasant - were presented by blocks), in order to induce a sustained emotional effect. This experiment was conducted on a separate sample $(N=43)$. The results showed no difference between MMN amplitudes recorded in emotional or neutral contexts, at any location; moreover, BIS- and BAS-related personality dimensions were not found to modulate the emotional context influence on MMN amplitude. Taken together with the above-reported experiment findings, this result suggests that personality might, indeed, influence automatic attention allocation to the unattended environment as a function of the context valence, but that this modulation is highly dependent on methodological factors, including the mode of context presentation.

In summary, the results of the present study did not replicate the findings of Surakka et al. (1998) and DePascalis et al. (2005) regarding emotional context influence on auditory MMN amplitude. On the other hand, the present results support the hypothesis that personality modulates the influence of emotional (namely unpleasant) context on attentional allocation to unattended environment (here indexed by MMN amplitude).

\section{Acknowledgments}

We thank Julie Depireux, François Drouguet, Kevin D’Ostilio, Aline Gillet, and Perrine Lardinois for their technical support and Christel Devue for her review of the manuscript.

\section{References}

Amrhein, C., Mühlberger, A., Pauli, P., \& Wiedemann, G. (2004). Modulation of event-related brain potentials during affective picture processing: A complement to startle reflex and skin conductance response? International Journal of Psychophysiology, 54, 231-240.

Bradley, B.P., \& Mogg, K. (1994). Mood and personality in recall of positive and negative information. Behavior Research and Therapy, 32, 137-141.

Canli, T., Sivers, H., Withfield, S.L., Gotlib, I.H., \& Gabrieli, J.D.E. (2002). Amygdala response to happy faces as a function of extraversion. Science, 296, 2191.

Carver, C.S., \& White, T.L. (1994). Behavioral inhibition, behavioral activation, and affective responses to impeding reward and punishment: The BIS/BAS scales. Journal of Personality and Social Psychology, 67, 319-333.

Center for the Study of Emotion and Attention [CSEA-NIMH]. (1999). International affective picture system: Digitalized photographs. Gainesville, FL: The Center for Research in Psychophysiology, University of Florida.

Cloninger, C.R. (1987). A systematic method for clinical description and classification of personality variants. Archives of General Psychiatry, 44, 573-588.

Cloninger, C.R. (1999). The temperament and character inventory-revised. St. Louis, MO: Center for Psychobiology of Personality, Washington University.

Cloninger, C.R., Svrakic, D.M., \& Przybeck, T.R. (1993). A psychobiological model of temperaments and character. Archives of General Psychiatry, 50, 975-990.

Corr, P.J. (2002). J.A. Gray's reinforcement sensitivity theory: Tests of the joint subsystem hypothesis of anxiety and impulsivity. Personality and Individual Differences, 33, 511-532.

Costa, P.T., \& McCrae, R.R. (1990). The NEO Personality Inventory manual revised. Odessa, FL: Psychological Assessment Resources.

DePascalis, V., Awari, B., Matteucci, A., \& Mazzocco, A. (2005). Effect of emotional visual stimuli on auditory information processing: A test of J.A. Gray's reinforcement sensitivity theory. Personality and Individual Differences, 38, 163-178.

DePascalis, V., \& Speranza, O. (2000). Personality effects on attentional shift to emotional charged cues: ERP, behavioral and HR data. Personality and Individual Differences, 29, 217-238.

DePascalis, V., Strippoli, E., Riccardi, P., \& Vergari, F. (2004). Personality, event-related potential (ERP), and hear rate (HR) in emotional word processing. Personality and Individual Differences, 36, 873-891.

Delplanque, S., Silvert, L., Hot, P., \& Sequeira, H. (2006). Eventrelated $\mathrm{P} 3 \mathrm{a}$ and $\mathrm{P} 3 \mathrm{~b}$ in response to unpredictable emotional stimuli. Biological Psychology, 68, 107-20.

Fabiani, M., Gratton, G., \& Coles, M.G.H. (2000). Event-related brain potentials: Methods, theory, and applications. In J.T. Cacioppo, L.G. Tassinary, \& G.G. Bernston (Eds.), Handbook of psychophysiology (2nd ed., pp. 53-84). New York: Cambridge University Press.

Franken, I.H., Nijs, I., \& VanStrien, J.W. (2005). Impulsivity affects mismatch negativity (MMN) measures of preattentive auditory processing. Biological Psychology, 70, 161-167.

Gable, S.L., Reis, H.T., \& Elliot, A.J. (2000). Behavioral activation and inhibition in everyday life. Journal of Personality and Social Psychology, 78, 1135-1149.

Gomez, A., \& Gomez, R. (2002). Personality traits of the behavioral approach and inhibition systems: Associations with processing of emotional stimuli. Personality and Individual Differences, 32, 1299-1316.

Gross, J.J., Sutton, S.K., \& Ketelaar, T. (1998). Relations between affect and personality: Support for the affect-level and affective-reactivity views. Personality and Social Psychology Bulletin, 24, 279-288.

Hansenne, M., Pinto, E., Scantamburlo, G., Renard, B., Reggers, J., Fuchs, S. et al. (2003). Harm avoidance is related to mismatch negativity (MMN) amplitude in healthy subjects. Personality and Individual Differences, 34, 1039-1048.

Heponiemi, T., Keltikangas-Järvinen, L., Puttonen, S., \& Ravaja, N. (2003). BIS/BAS sensitivity and self-rated affects during 
experimentally induced stress. Personality and Individual Differences, 34, 943-957.

Lang, P.J., Greenwald, M.K., Bradley, M.M., \& Hamm, A.O. (1993). Looking at pictures: Affective, facial, visceral, and behavioral reactions. Psychophysiology, 30, 261-273.

Näätänen, R. (2000). Mismatch negativity (MMN): Perspectives for application. International Journal of Psychophysiology, 37, 3-10.

Näätänen, R. (2001). The perception of speech sounds by the human brain as reflected by the mismatch negativity (MMN) and its magnetic equivalent (MMNm). Psychophysiology, 38, 1-21.

Näätänen, R., Gaillard, A.W., \& Mantysalo, S. (1978). Early selective-attention effect on evoked potential reinterpreted. Acta Psychologica, 42, 313-329.

Palomba, D., Angrilli, A., \& Mini, A. (1997). Visual evoked potentials, heart rate responses, and memory to emotional pictorial stimuli. International Journal of Psychophysiology, 27, $55-67$.

Picton, T.W., Alain, C., Otten, L., Ritter, W., \& Achim, A. (2000). Mismatch negativity: Different water in the same river. Audiology and Neurootology, 5, 111-139.

Rusting, C.L. (1999). Interactive effects of personality and mood on emotion-congruent memory and judgment. Journal of Personality and Social Psychology, 77, 1073-1086.

Sasaki, T., Campbell, K.B., Gordon Bazana, P., \& Stelmack, R.M. (2000). Individual differences in mismatch negativity measures of involuntary attention shift. Clinical Neurophysiology, $111,1553-1560$.

Surakka, V., Tenhunen-Eskelinen, M., Hietanen, J.K., \& Sams, M. (1998). Modulation of human auditory information processing by emotional visual stimuli. Cognitive and Brain Research, 7, 159-163.
Torrubia, R., Avila, C., Moltó, J.M., \& Caseras, X. (2001). The Sensitivity to Punishment and Sensitivity to Reward Questionnaire (SPSRQ) as a measure of Gray's anxiety and impulsivity dimensions. Personality and Individual Differences, 31, 837-862.

Van de Velde, M., Ghosh, I.R., \& Cluitmans, P.J. (1999). Contextrelated artefact detection in prolonged EEG recordings. Computer Methods and Programs in Biomedicine, 60, 183-196.

Wang, W., Zhu, S.Z., Pan L.C., Hu, A.H., \& Wang, Y.H. (2001). Mismatch negativity and personality traits in chronic primary insomniacs. Functional Neurology, 16, 3-10.

Winkler, I. \& Cowan, N. (2005). From sensory to long-term memory: Evidence from auditory memory reactivation studies. $E x$ perimental Psychology, 52, 3-20.

Winkler, I., Cowan, N., Csépe, V., Czigler, I., \& Näätänen, R. (1996). Interactions between transient and long-term auditory memory as reflected by the mismatch negativity. Journal of Cognitive Neuroscience, 8, 403-415.

Solange Mardaga

Faculty of Psychology

Department of Cognitive Sciences

University of Liège

5, boulevard du Rectorat (B32)

B-4000 Liège

Belgium

Tel. +3243663320

Fax +32 43662944

E-mail solange.mardaga@student.ulg.ac.be 